\title{
Photoresponsive Permeation Characteristics of a Ternary Composite Membrane of Polymer/Artificial Lipid/Azobenzene Derivative
}

\author{
Atsushi Kumano, Osamu Niwa, Tisato Kajiyama, ${ }^{\dagger}$ \\ Motowo TAKAYANAGI, Toyoki KunitaKe, ${ }^{*}$ \\ and Koji KANO** \\ Department of Applied Chemistry and *Organic Synthesis, \\ Faculty of Engineering, Kyushu University, \\ 6-10-1, Hakozaki, Higashi-ku, \\ Fukuoka 812, Japan \\ ${ }^{* *}$ Faculty of Engineering, Doshisha University, \\ Kyoto 602, Japan
}

(Received December 27, 1983)

\begin{abstract}
A ternary composite membrane composed of polymer/artificial lipid/azobenzene derivative was prepared by casting from a tetrahydrofuran solution of poly(vinyl chloride) (PVC), dioctadecyldimethylammonium bromide (DOAB) and $p$ - $\omega$-hydroxypropyloxy- $p^{\prime}$-dodecyloxy azobenzene (AZO). AZO dispersed almost molecularly within the multilayer liposome of artificial lipid (DOAB), and even in the ternary composite membrane, AZO exhibited trans-cis photoisomerization in a similar manner to that in solution. The water or ion permeations through the composite membrane apparently increased upon the irradiation of ultraviolet (UV) light when artificial lipids were in a liquid crystalline state. The water permeability coefficient, $P$ depended on the trans-fraction of AZO. This suggests that the conformational change due to the trans-cis photoisomerization of AZO makes compact packing of artificial lipid molecules around AZO loosed, being followed by formation of permeation channel.

KEY WORDS Ternary Composite Membrane / Poly(vinyl chloride) / Artificial Lipid / Dioctadecyldimethylammonium Bromide / Azobenzene Derivative / Photoisomerization / Thermal Isomerization / Photoresponsive Permeation / Permeation /
\end{abstract}

Lipids in biological systems are amphiphiles composed of both hydrophobic group of two alkyl chains and hydrophilic group of phospholyl group. Biological membranes are in a liquid crystalline state on which their permeation properties depend. ${ }^{1,2}$ One of authors has succeeded in synthesis of totally artificial lipids as a biomembrane model. ${ }^{3-5}$ The artificial lipid/water system showed the similar phase diagram to that of biological lipids. ${ }^{6}$ The composite membrane composed of polymer and artificial lipid exhibited a distinct jump of

\footnotetext{
$\dagger$ To whom correspondence should be addressed
}

the water and ion permeability coefficients in the vicinity of crystal-liquid crystal phase transition temperature. ${ }^{7}$ These results apparently indicate that the diffusion process of permeable substances through the membrane is closely related to the thermal molecular motion of lipid molecules.

Photoresponsive systems are well known in nature. ${ }^{8}$ Some of them have their primary biochemical response to light converted into a physiological process by means of a complex pathway. For example, 11-cis-retinal, the chromophore of rhodopsin, undergoes a cis to trans isomerization upon irradiation of 


\section{A. Kumano et al.}

light. ${ }^{9,10}$ It has been reported that the light activation of rhodopsin/phospholipid liposomes leads to an increase of membrane permeation. ${ }^{11-13}$ Also, photoresponsive amphiphiles such as azobenzene derivatives were synthesized as a biological lipid model. ${ }^{14}$ They have been studied from the viewpoint of permeation control for phospholipid/azobenzene derivative liposomes. ${ }^{15}$ These trans-azobenzene derivatives have a characteristic of transformation to cis-isomers upon an irradiation of wavelength of the $360 \mathrm{~nm}$ light, and the latter to the former upon an irradiation of light above wavelength of $420 \mathrm{~nm}$ or heating. In the phospholipid liposomes incorporating azobenzene derivatives, the conformational change from a trans- to a cis-isomers induces the formation of a wide permeation pathway due to the local disorder of chain packing. Therefore, the membrane permeability increased by the irradiation of ultraviolet (UV) light of $360 \mathrm{~nm} \cdot{ }^{15}$ Also, the facilitated photoresponsive transport of metal cation has been studied for the polymer/liquid crystal/azobenzene-bridged crown ether ternary composite membrane. This system is fairly good example of the carrier mechanism for metal cation transport. ${ }^{16}$

Though unilamellar or multilamellar liposomes in solution are a useful biomimetic system for permeation study in a laboratory, they are not advantageous for engineering application, because both mechanical stabilities of liposomes and a thin film with twodimensional large area are required. In order to prepare the wide and thin artificial membrane having characteristics of biological membrane, the blend systems of polymer and artificial lipids have been studied on the basis of permeation properties. The purpose of this paper is to investigate the water or ion permeation characteristics through a ternary composite membrane of polymer/artificial lipid/ azobenzene derivative, which are controlled by the trans-cis photoisomerization of azobenzene derivative.

\section{EXPERIMENTAL}

\section{Materials}

The chemical structures of polymer, artificial lipid and azobenzene derivative used are shown in Figure 1. Poly(vinyl chloride) (PVC) was chosen as the matrix substance for the ternary composite membrane because it does not exhibit any apparent thermal transition in the temperature range studied here. PVC was Vinyca 37L (Mitsubishi Monstant Kasei Co., Ltd.) of which number average molecular weight was 66000 . Dioctadecyldimethylammonium bromide (DOAB) of artificial lipid was synthesized by stepwise alkylation of dimethylamine. $p$ - $\omega$-Hydroxypropyloxy- $p^{\prime}$-dodecyloxyazobenzene (AZO), the photoresponsive substance, was synthesized by diazo coupling of $p$-(dodecyloxy)aniline and phenol and also, by subsequent reaction with 3bromopropanol.

A composite membrane of PVC/DOAB/ AZO was prepared by casting a $8 \mathrm{wt} \%$ tetrahydrofuran (THF) solution of PVC, DOAB, and AZO on the glass plate at $313 \mathrm{~K}$ for one day. Then, it was dried in vacuo for one week at room temperature. The thickness of the composite membrane was $15-30 \mu \mathrm{m}$. The weight percentages of $\mathrm{DOAB}$ and $\mathrm{AZO}$ were $13.1 \mathrm{wt} \%$ and $1.9 \mathrm{wt} \%$, respectively, and their molar ratio was $\mathrm{DOAB} / \mathrm{AZO}=5 / 1$.

\section{Characterization of Ternary Composite Mem- brane}

Differential scanning calorimetry (DSC) and $\mathrm{X}$-ray studies were performed in order to investigate the crystal-liquid crystal phase transition behavior of $\mathrm{DOAB}$ and a dispersing state of AZO molecules in the composite membrane. Also, transmission electron microscopic observation (TEM) of PVC/DOAB (85/15 in $\mathrm{wt}^{\mathrm{o}} \%$ ) thin film was performed in order to investigate the aggregation state of DOAB in the composite membrane. A $0.1 \mathrm{wt} \% \mathrm{THF}$ solution of PVC and DOAB was dropped on a carbon grid and dried for TEM observation. 
Photoresponsive Permeation of Composite Membrane

1 POLYMER

POLY (VINYL CHLORIDE) (PVC)

$\left.+\underset{\mathrm{Cl}}{\mathrm{CHCH}_{2}}\right)_{\sqrt[n]{n}}$

2 ARTIFICIAL LIPID

DIOCTADECYLDIMETHYLAMMONI UM BROMIDE (DOAB)

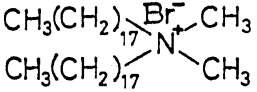

3 AZOBENZENE DERIVATIVE

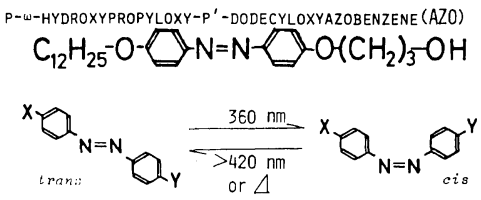

Figure 1. Chemical structures of components for the composite membrane.

Subsequently, its surface was treated by ionetching for $10 \mathrm{~s}$ in order to perform effective staining. Then, the composite membrane was negatively stained with a $0.05 \mathrm{wt} \%$ aqueous solution of uranyl acetate.

The composite membrane was irradiated with the light of wavelength of $360 \mathrm{~nm}$ for several minutes in order to induce a trans-cis isomerization of AZO within the membrane. The light source was a Toshiba $100 \mathrm{~W}$ super high-pressure $\mathrm{Hg}$ lamp. Spectral change of trans-AZO in the composite membrane upon UV irradiation was obtained using JASCO 505 spectrophotometer.

\section{Permeation Study through Ternary Composite Membrane}

The water and $\mathrm{K}^{+}$permeabilities were measured by a normal osmosis method using a $\mathrm{KCl}$ aqueous solution of $1 \mathrm{~mol} \mathrm{dm}^{-3}$ and distilled water separated by the composite membrane. Figure 2 shows a permeation cell used in this work. The quartz glass plate was set on the one end of the cell in order to irradiate the UV light perpendicular to the membrane surface. An effective membrane area was $9.0 \mathrm{~cm}^{2}$. The quantity of permeated water through the composite membrane was evaluated from the variation of water level

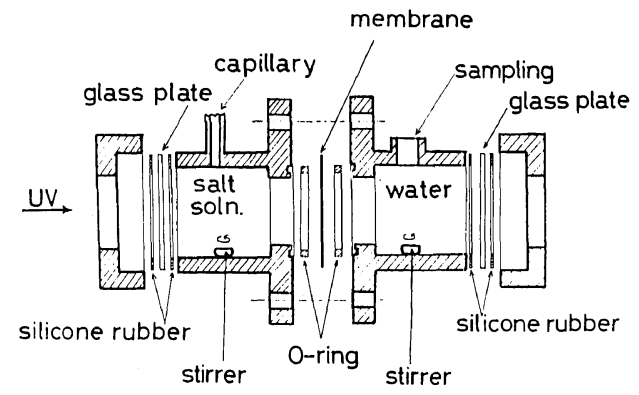

Figure 2. Permeation cell for photoresponsive study.

height in the capillary. The flux of $\mathrm{K}^{+}$was evaluated by measuring the time dependence of $\mathrm{K}^{+}$concentration in the downstream side with an atomic absorption spectrophotometer. The UV light was irradiated perpendicular to the membrane surface and the distance between the light source and the membrane surface was $140 \mathrm{~mm}$.

\section{RESULTS AND DISCUSSION}

\section{Characterization of Ternary Composite Mem- brane}

Figure 3 shows the $\mathrm{X}$-ray diffraction photograph of the $\mathrm{PVC} / \mathrm{DOAB} / \mathrm{AZO}$ composite membrane. The incident $\mathrm{X}$-ray beam was perpendicular to the membrane surface. The diffraction pattern apparently indicates the existence of DOAB crystallite on the basis of the magnitude of diffraction spacings. The long spacing of $2.98 \mathrm{~nm}$ for the PVA/DOAB/AZO composite membrane corresponds to that of $2.8 \mathrm{~nm}$ for DOAB/AZO (5/1) mixture. Though these magnitudes of the long spacing were different from those of DOAB $(3.6 \mathrm{~nm})$ and AZO $(5.6 \mathrm{~nm})$ themselves, it seems apparent that there exists a layer structure of $\mathrm{DOAB} /$ AZO in the PVC matrix since a ratio of the reciprocals of the long spacings for the 1 st and 2nd order scatterings is $1: 2$.

Figure 4 shows the transmission electron micrographs for the blend thin film of PVC/ DOAB, which was negatively stained with uranyl acetate. In the magnified area (right photograph), the layer structure was ap- 


\section{A. Kumano et al.}

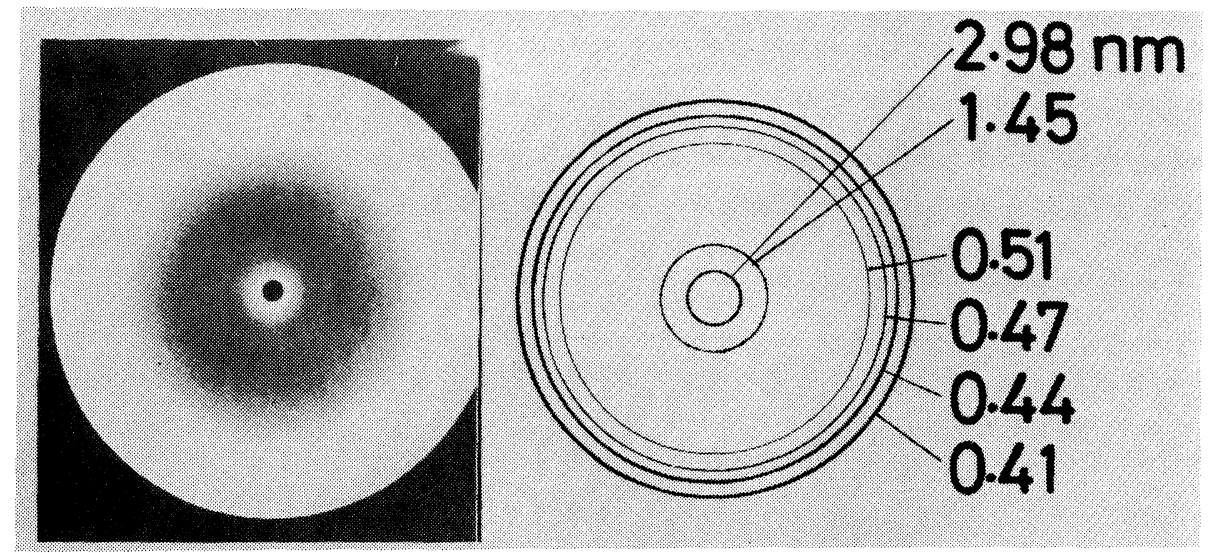

Figure 3. X-Ray diffraction photograph and its schematic representation of PVC/DOAB/AZO composite membrane.
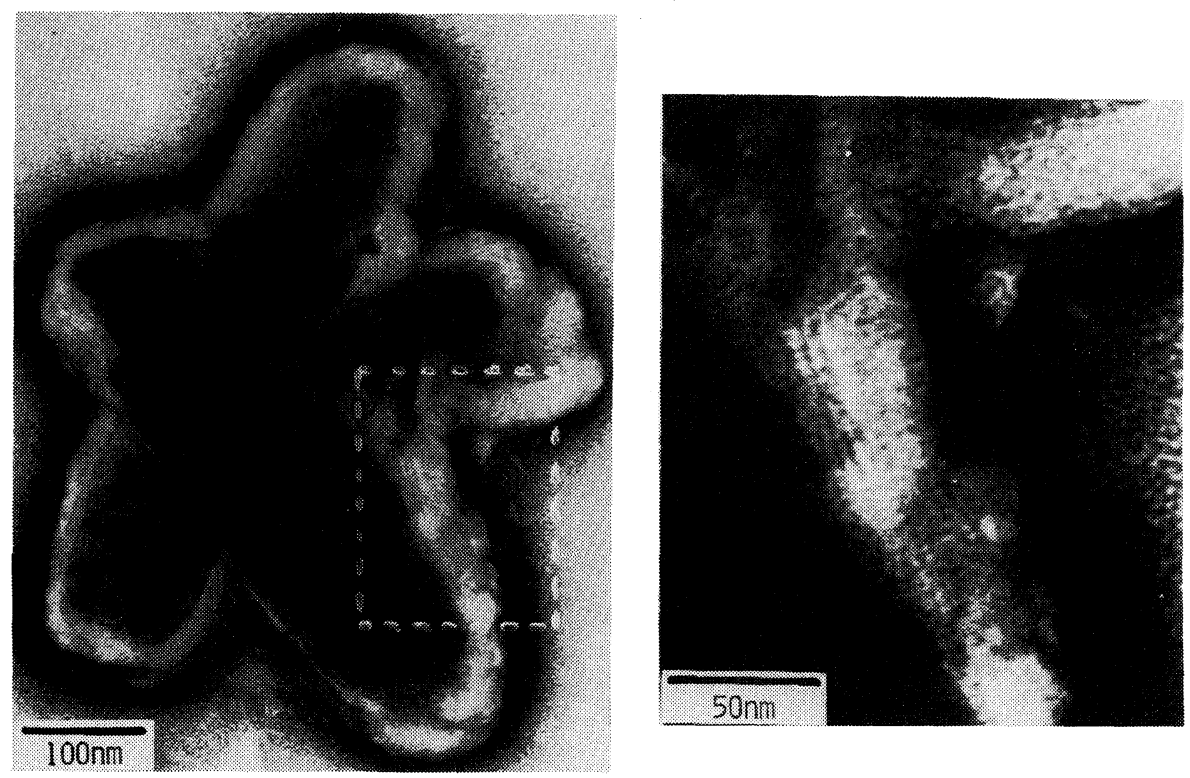

$\mathrm{PVC} / \mathrm{DOAB}=85 / 15$

Figure 4. Transmission electron micrograph of PVC/DOAB composite membrane (left) and enlarged one of selected area in the left photograph encircled by broken lines (right). The composite membrane is negatively stained with uranyl acetate.

parently observed. The spacing between dark striations was $4.0-4.6 \mathrm{~nm}$ and was consistent with the mean thickness of bilayer of DOAB. ${ }^{6}$ This result indicates that DOAB multilayer structure exists even in the polymer matrix.

Figure 5 shows the DSC curves of DOAB, $\mathrm{AZO}, \mathrm{DOAB} / \mathrm{AZO}(5 / 1$ in molar ratio) and the composite membrane. DOAB (dry) exhibited the endothermic peak corresponding to the crystal (K)-liquid crystal (Meso) phase transition, $T_{\mathrm{K} \rightarrow \mathrm{M}}$ at $357 \mathrm{~K}$. The melting temperature of AZO was $399 \mathrm{~K}$. The phase transition temperature of $\mathrm{DOAB}$ in the $\mathrm{DOAB} /$ AZO mixture (dry) shifted to lower tempera- 
ture compared with that of DOAB itself (from the case (1) to the case (3)) and furthermore, the melting behavior of AZO disappeared (from the case (2) to the case (3)). These DSC results indicate that $\mathrm{AZO}$ molecules disperse almost in a molecular level within the multilayer of DOAB. $T_{\mathrm{K} \rightarrow \mathrm{M}}$ of DOAB in the PVC/ DOAB/AZO composite membrane was $343 \mathrm{~K}$ in case of a dry condition and was depressed to $317 \mathrm{~K}$ in case of a wet condition. This result suggests that the polar head group of artificial lipids was bound to water, even though in the composite membrane. $T_{\mathrm{K} \rightarrow \mathrm{M}}$ of DOAB (dry) in the ternary composite membrane became

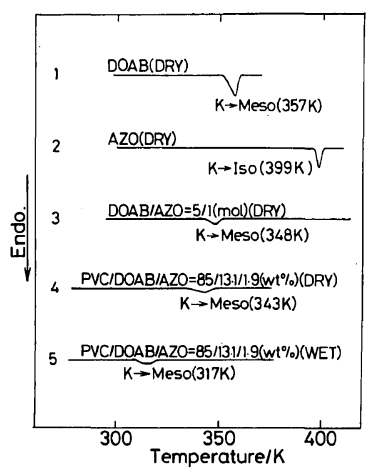

Figure 5. DSC curves of DOAB, AZO, DOAB/AZO mixture and composite membrane. $\mathrm{K}$, Meso and Iso designate crystal phase, mesophase and isotropic phase, respectively. slightly lower than that for the DOAB/ AZO system (from the case (3) to the case (4)). This slight depression of $T_{\mathrm{K} \rightarrow \mathrm{M}}$ may suggest that $\mathrm{DOAB}$ in the composite membrane aggregates in a relatively unstable state such as the reverse bimolecular aggregation, in which the hydrophobic portion of hydrocarbon chains faces to the outer direction in the outermost layer of DOAB multilayer and contacts with the polymer matrix. This type of aggregation may be easily formed due to considerable affinity between the hydrophobic portion of artificial lipid and polymer.

Photo- and Thermal Isomerizations of AZO in Composite Membrane

Photo- and thermal isomerizations of AZO in the composite membrane was studied on the basis of the UV spectroscopic measurement. The composite membrane was interposed between the quartz glass plates and set in the spectrophotometer. Figure 6 shows the spectral change of the ternary composite membrane upon irradiation of the $360 \mathrm{~nm}$ UV light at $283 \mathrm{~K}$. At this temperature, DOAB molecules were in a crystalline state (below $T_{\mathrm{K} \rightarrow \mathrm{M}}$ ). The absorption maximum of trans-AZO was observed at $360 \mathrm{~nm}$. The peak height decreased with an increase in irradiation time of the $360 \mathrm{~nm}$ UV light. On the other hand, the

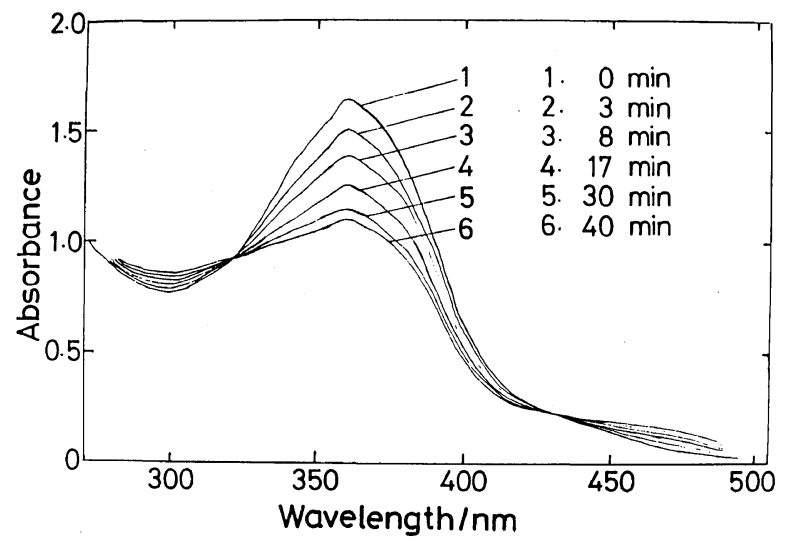

Figure 6. Spectral change of the ternary composite membrane as a function of irradiation time of $360 \mathrm{~nm}$ light at $283 \mathrm{~K}$. 


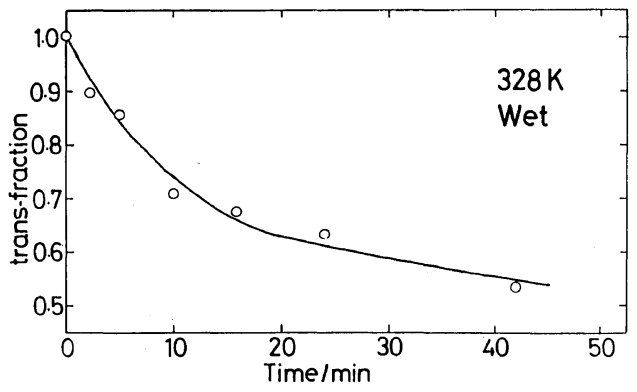

Figure 7. Decrease in trans-fraction of the ternary composite membrane as a function of irradiation time of the $360 \mathrm{~nm}$ UV light at $328 \mathrm{~K}$.

absorption intensity around $300 \mathrm{~nm}$ and $470 \mathrm{~nm}$ increased due to the concomitant formation of cis-AZO. This means that the transcis photoisomerization of AZO molecule takes place even in the ternary composite membrane similarly to the case in solution. Since the cisisomer is more bulky than the trans-isomer, ${ }^{17}$ the regular alignment or compact packing of DOAB molecules may be disordered by the trans-cis isomerization of AZO derivatives which are molecularly dispersed in the bimolecular layer of DOAB. Also, in the case of a liquid crystalline state of DOAB above $T_{\mathrm{K} \rightarrow \mathrm{M}}$, the trans-cis photoisomerization behavior was observed in the similar manner to that below $T_{\mathrm{K} \rightarrow \mathrm{M}}$ mentioned above.

Figure 7 shows the trans-AZO fraction as a function of irradiation time of the $360 \mathrm{~nm} \mathrm{UV}$ light at $328 \mathrm{~K}$ (above $T_{\mathrm{K} \rightarrow \mathrm{M}}$ ). Figure 7 was obtained by using the ternary composite membrane in the wet condition, which was a similar condition to permeation experiment. The trans-fraction was evaluated from the change of the absorption peak height at $360 \mathrm{~nm}$. The base line was drawn between 300 and $430 \mathrm{~nm}$ on the spectrum of curve 1 in Figure 6, taking polymer scattering and overlapping of cisAZO absorption into consideration. The peak height from the base line at $360 \mathrm{~nm}$ before irradiation of the UV light was assumed to be equal to a unity. Figure 7 tells us that the isomerization reaches gradually to the equilib-

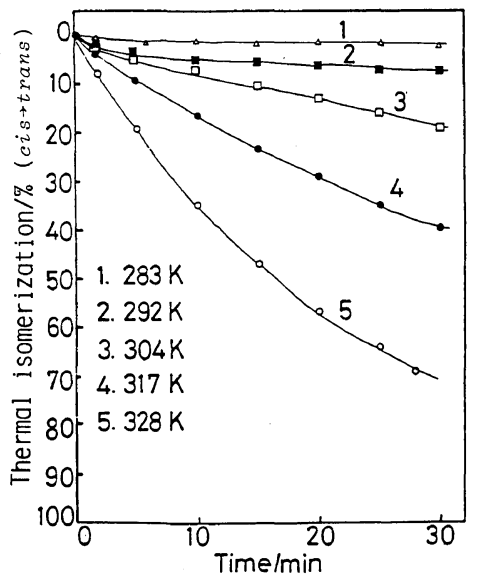

Figure 8. Time dependence of thermal isomerization from cis- to trans-AZO in the dark as a function of measuring temperature.

rium state at about $50-60 \%$ of the transfraction due to the cis $\rightarrow$ trans reverse transformation.

In order to investigate the photoresponsive control of the water or ion permeation for the ternary composite membrane, the temperature dependence of the thermal isomerization rate must be considered, because the thermal isomerization as well as the photoisomerization is caused at a higher temperature during irradiation of the UV light. The thermal isomerization reaction of $\mathrm{AZO}$ in the composite membrane was evaluated by measuring the time dependence of the absorption maximum at $360 \mathrm{~nm}$ in the dark. The composite membrane was irradiated by the $360 \mathrm{~nm}$ light up to attaining the equilibrium on the trans $\rightarrow$ cis photoisomerization and then, was left in the dark at several temperatures as shown in Figure 8. Figure 8 shows the temperature dependence of thermal isomerization of AZO from the cis to trans form. The percentage of thermal isomerization was determined by the magnitude of reincrease in the absorption peak at $360 \mathrm{~nm}$ in the dark. As shown by the curve 1 in Figure 8, the thermal isomerization could be neglected below $283 \mathrm{~K}$ since it was so slow in comparison with the photoisomerization. The rate of ther- 
mal isomerization was considerably fast at around $328 \mathrm{~K}$ and this was an inevitable reverse reaction upon the photoisomerization above $328 \mathrm{~K}$. A plot of the logarithm of the cis-fraction against the thermal isomerization time exhibited a farily good first-order reaction. This reaction rate constant, $k$ was $3.08 \times 10^{-4} \mathrm{~s}^{-1}$ at $328 \mathrm{~K}$. Since a certain fraction of cis-AZO transforms to trans-AZO due to the thermal isomerization after light off upon permeation experiment, the true fraction of trans-AZO was evaluated by using the value of $k$.

Water or $K^{+}$Permeation through Photoresponsive Ternary Composite Membrane

Figures 9 and 10 show the photoresponsive effect on the time dependences of the permeation quantity of water, $q$ at $328 \mathrm{~K}$ and $\mathrm{K}^{+}$ concentration in the downstream side at $321 \mathrm{~K}$, respectively, for the $\mathrm{PVC} / \mathrm{DOAB} / \mathrm{AZO}$ ternary composite membrane. As mentioned before, the DOAB/AZO mixture was in a liquid crystalline state in the composite membrane at these temperatures. The UV irradiation period was $30 \mathrm{~min}$ for water permeation experiment and $60 \mathrm{~min}$ for $\mathrm{K}^{+}$one, respectively, as indicated by light on and light off. Permeation of water and $\mathrm{K}^{+}$were apparently accelerated upon irradiation of the $360 \mathrm{~nm}$ light when the DOAB/AZO mixture was in a liquid crystalline state. Since $c i s-A Z O$ is more bulky than trans-AZO, it is reasonable to consider that the DOAB multilayer becomes more disordered state with an increase in the cis-AZO fraction due to the trans $\rightarrow$ cis photoisomerization. Therefore, the apparent increase in permeability to water or $\mathrm{K}^{+}$may be explained by an increase in the number and/or the fraction of effective channel or vacancy for permeation.

On the other hand, in the case below $T_{\mathrm{K} \rightarrow \mathrm{M}}$, the permeation of water or $\mathrm{K}^{+}$was not detectably affected by irradiation of the $360 \mathrm{~nm}$ light, though the trans-cis isomerization certainly occurred. Generally speaking, water

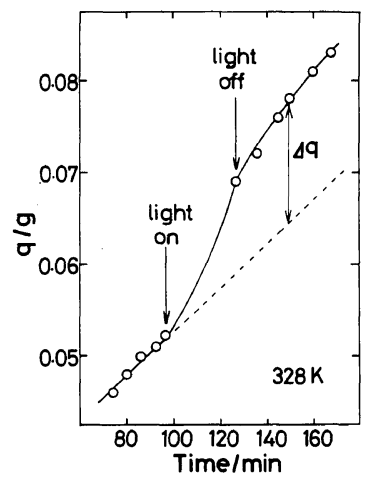

(a)

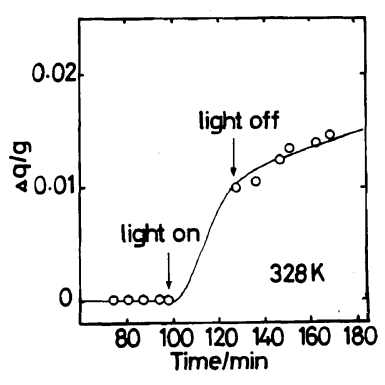

(b)

Figure 9. (a): Change in quantity of water permeation, $q$ at $328 \mathrm{~K}$ affected by light on or off. (b): Photoisomerization effect on relative water permeation, $\Delta q$ at $328 \mathrm{~K}$.

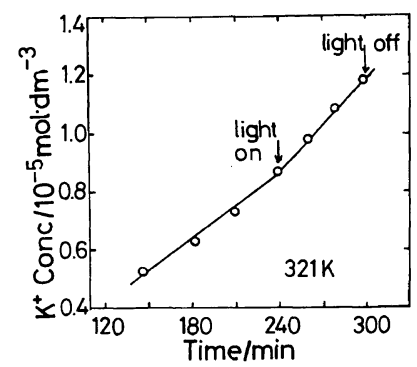

Figure 10. Change in quantity of $\mathrm{K}^{+}$permeation at $321 \mathrm{~K}$ affected by light on.

molecules or $\mathrm{K}^{+}$can not diffuse through the DOAB multilayer in a crystalline state. Though the molecular arrangement in a crystalline state may be locally disordered by the trans-cis photoisomerization, possibility of the continuous vacancy passing through the DOAB multilayer seems to be very low. 


\section{A. Kumano et al.}

Therefore, the permeabilities to water and $\mathrm{K}^{+}$ did not apparently change by the irradiation of the $360 \mathrm{~nm}$ UV light below $T_{\mathrm{K} \rightarrow \mathrm{M}}$. These results of permeation experiment indicate that the permeation properties are strongly dependent upon the phase transition behaviors of artificial lipids and also, the permeabilities to water and $\mathrm{K}^{+}$are remarkably facilitated upon the irradiation of the UV light. Accordingly, it is reasonable to consider that the DOAB/AZO forms the continuous domain within the composite membrane and also, water or ion passes mainly through such continuous domain in a liquid crystalline state.

Indeed, the various types of the aggregation states of DOAB multilayer may coexist in the composite membrane. It is considered as extreme examples that the bilayer surface of DOAB aggregates parallel or perpendicular to the composite membrane surface resulting in formation of the multilayer structure. Though the aggregation and dispersing states of DOAB multilayer have not been investigated in detail, it is undoubted from the temperature dependence of the photoresponsive permeation that DOAB multilayer effectively contributes to water or $\mathrm{K}^{+}$permeation in the ternary composite membrane.

The value of the permeation rate, $\mathrm{d} q / \mathrm{d} t$ gradually decreased with time after turning off the $360 \mathrm{~nm}$ UV light as shown in Figure 9(a). After turning off the light, the fraction of trans-AZO increased again with time, because cis-AZO transformed thermally to trans-AZO at $328 \mathrm{~K}$. This behavior is resulted in a decrease in channel or vacancy in the DOAB multilayer, in other words, an increase in the ordered region of DOAB molecular chain packing. Consequently, the rate of water permeation decreased gradually with time after light off and it approached to that before irradiation of the UV light.

As discussed above, the permeability to water or $\mathrm{K}^{+}$through the ternary composite membrane was affected by the packing state of DOAB molecules, in other words, the fraction

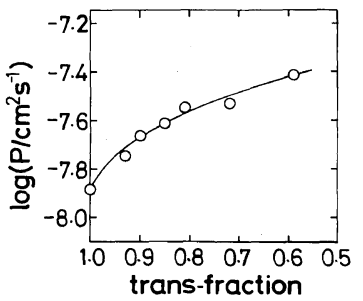

Figure 11. Change in diffusive permeability coefficient, $P$ to water with trans-fraction of AZO.

of trans-AZO. Figure 11 shows a plot of the diffusive permeability coefficient, $P$ to water against the trans-AZO fraction. The transfraction was evaluated by using the data in Figure 7 and the value of $k$. Upon photoisomerization, the trans-fraction reached to 0.59 at $30 \mathrm{~min}$ after irradiation at $360 \mathrm{~nm}$ (Figure 7). This experimental value of 0.59 corresponded to the simultaneous processes from photoisomerization and thermal isomerization during irradiation of the UV light at $328 \mathrm{~K}$. The degree of the reverse reaction from cis-AZO to trans-AZO induced by thermal isomerization after turning off the UV light at $328 \mathrm{~K}$ was calculated by the rate constant, $k$. Also, the variation of $P$ was evaluated by the slope of the permeation curve in Figure 9(a). As shown in Figure 11, the magnitude of $P$ decreased with an increase in the trans-AZO fraction and, in other words, water diffusion through the DOAB multilayer became easily with an increase in the cis-AZO fraction. Therefore, Figure 11 indicates that the trans $\rightarrow$ cis isomerization of AZO may induce disordering of compact packing of DOAB molecules around AZO, resulting in an increase in number of channel or vacancy for water permeation in the multilayer of DOAB.

The permeation mechanism of the photoresponsive ternary composite membrane above $T_{\mathrm{K} \rightarrow \mathrm{M}}$ could be schematically explained as shown in Figure 12 on the basis of the photoresponsive characteristic permeation of water and $\mathrm{K}^{+}$. When AZO molecules have the transconformation, DOAB bilayer can retain the 


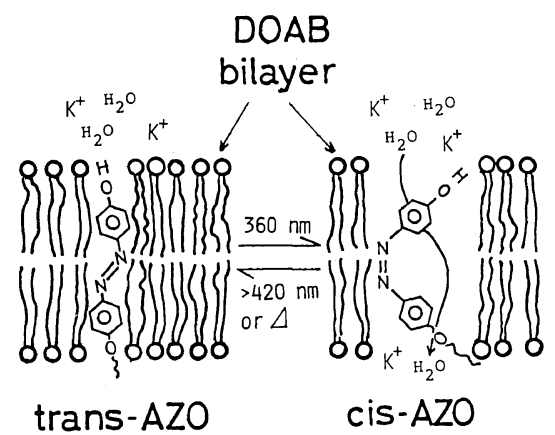

Figure 12. Schematic representation of formation of channel in composite membrane due to local disordering of $\mathrm{DOAB}$ molecular aggregation.

compact packing. Though permeable substances can pass through the fluid region of DOAB bilayer above $T_{\mathrm{K} \rightarrow \mathrm{M}}$, its permeability is not prominent because of considerable compact packing of DOAB molecules. On the other hand, when AZO molecules transform to cis-conformation by irradiation of the UV light, the transmembrane channels may be formed in the DOAB bilayer due to bulkiness of the cis-AZO as shown in Figure 12. Therefore, it is apparent that permeable substances pass through the composite membrane with the higher fraction of cis-AZO more easily than that with trans-AZO conformation. Consequently, the permeability to water or $\mathrm{K}^{+}$ for the ternary composite membrane above $T_{\mathrm{K} \rightarrow \mathrm{M}}$ increased significantly by irradiation of the UV light, according to the postulated mechanism shown in Figure 12.

\section{CONCLUSION}

From DSC, X-ray and morphological studies, it was concluded that the DOAB/ AZO mixture was capable of formation of multilayer structure in the polymer/DOAB/ AZO ternary composite membrane, and AZO molecule dispersed almost monomerically in the $\mathrm{DOAB}$ multilayer. The aggregation state of DOAB molecules was controlled by the conformational change of AZO molecules.
AZO molecules in the composite membrane exhibited photo- or thermal isomerizations upon irradiation of the $360 \mathrm{~nm}$ UV light or heating, respectively, in the similar manner to that in solution.

The permeability of the composite membrane to water or $\mathrm{K}^{+}$increased by irradiation of the $360 \mathrm{~nm}$ UV light in the case of the liquid crystalline state of DOAB (above $T_{\mathrm{K} \rightarrow \mathrm{M}}$ ). The detectable increase of water or $\mathrm{K}^{+}$permeation through the ternary composite membrane has not been observed by irradiation of the UV light below $T_{\mathrm{K} \rightarrow \mathrm{M}}$. It was concluded from these results that the transmembrane channels were effectively formed by the trans $\rightarrow$ cis photoisomerization of AZO upon irradiation of the $360 \mathrm{~nm}$ UV light when DOAB was in a liquid crystalline state, and water or $\mathrm{K}^{+}$permeated along these channels through the DOAB multilayers. Therefore, permeable substances became more easily to transfer across the DOAB bilayer membranes accompanied by cisconformation of AZO.

\section{REFERENCES}

1. M. C. Block, L. L. M. van Deenen, and J. DeGier, Biochim. Biophys. Acta, 443, 1 (1976).

2. K. Inoue, Biochim. Biophys. Acta, 339, 390 (1974).

3. T. Kunitake, J. Macromol. Sci. Chem., A13, 587 (1979).

4. Y. Okahata and T. Kunitake, J. Am. Chem. Soc., 101, 5231 (1979).

5. T. Kunitake and Y. Okahata, J. Am. Chem. Soc., 102, 549 (1980).

6. T. Kajiyama, A. Kumano, M. Takayanagi, Y. Okahata, and T. Kunitake, Contemporary Topics in Polym. Sci., 4, 829 (1984).

7. T. Kajiyama, A. Kumano, M. Takayanagi, Y. Okahata, and T. Kunitake, Chem. Lett., 645 (1977). A. Kumano, T. Kajiyama, M. Takayanagi, and T. Kunitake, Rep. Prog. Polym. Phys. Jpn., 25, 793 (1982).

8. B. F. Erlanger, Ann. Rev. Biochem., 5, 267 (1976).

9. G. Wald, Nature (London), 219, 800 (1968).

10. T. G. Ebrey and B. Honig, Q. Rev. Biophys., 8, 129 (1975).

11. A. Darszon, M. Montal, and J. Zarco, Biochem. Biophys. Res. Commun., 76, 820 (1977).

12. D. F. O'Brien, N. Zumbulyadis, F. M. Michaels, and R. A. Ott, Proc. Natl. Acad. Sci. U.S.A., 74, 5222 
A. Kumano et al.

(1977).

13. D. F. O'Brien, Photochem. Photobiol., 29, 679 (1979).

14. T. Kunitake, Y. Okahata, M. Shimomura, S. Yasunami, and T. Takarabe, J. Am. Chem. Soc., 103, 5401 (1981)

15. K. Kano, T. Tanaka, T. Ogawa, M. Shimomura, and
T. Kunitake, Photochem. Photobiol., 34, 323 (1981).

16. A. Kumano, O. Niwa, T. Kajiyama, M. Takayanagi,

K. Kano, and S. Shinkai, Chem. Lett., 1327 (1983).

17. G. C. Hampson and J. M. Robertson, J. Chem. Soc., 409 (1941). 